\title{
Prospective cohort study for identification of underlying genetic causes in neonatal encephalopathy using whole-exome sequencing
}

\author{
Theodora U.J. Bruun, BSc ${ }^{1,2}$, Caro-Lyne DesRoches, $\mathrm{MSc}^{1}$, Diane Wilson, BScN, BN ${ }^{3,4}$, Vann Chau, MD ${ }^{4}$, \\ Tadashi Nakagawa, $\mathrm{PhD}^{5}$, Masahiro Yamasaki, $\mathrm{PhD}^{6}$, Shinya Hasegawa, $\mathrm{PhD}^{6}$, \\ Toshiyuki Fukao, MD, $\mathrm{PhD}^{7}$, Christian Marshall, $\mathrm{PhD}^{1,8}$ and \\ Saadet Mercimek-Andrews, MD, PhD ${ }^{1,2,9}$
}

\begin{abstract}
Purpose: Neonatal encephalopathy, which is characterized by a decreased level of consciousness, occurs in 1-7/1,000 live-term births. In more than half of term newborns, there is no identifiable etiological factor. To identify underlying genetic defects, we applied whole-exome sequencing (WES) in term newborns with neonatal encephalopathy as a prospective cohort study.

Methods: Term newborns with neonatal encephalopathy and no history of perinatal asphyxia were included. WES was performed using patient and both parents' DNA.

Results: Nineteen patients fulfilling inclusion criteria were enrolled. Five patients were excluded owing to withdrawal of consent, no parental DNA samples, or a genetic diagnosis prior to WES. Fourteen patients underwent WES. We confirmed a genetic diagnosis in five patients (36\%): epileptic encephalopathy
\end{abstract}

associated with autosomal dominant de novo variants in SCN2A (p.Met1545Val), KCNQ2 (p.Asp212Tyr), and GNAO1 (p.Gly40Arg); lipoic acid synthetase deficiency due to compound heterozygous variants in LIAS (p.Ala253Pro and p.His236Gln); and encephalopathy associated with an X-linked variant in CUL4B (p.Asn211Ser).

Conclusion: WES is helpful at arriving genetic diagnoses in neonatal encephalopathy and/or seizures and brain damage. It will increase our understanding and probably enable us to develop targeted neuroprotective treatment strategies.

Genet Med advance online publication 17 August 2017

Key Words: genetic; hypoxic-ischemic encephalopathy; neonatal encephalopathy; neonatal seizures; whole-exome sequencing

\section{INTRODUCTION}

Neonatal encephalopathy results from a dysfunction of the central nervous system in term newborns and is characterized by a decreased level of consciousness, seizures, respiratory insufficiency, and depression of tone and reflexes. Neonatal encephalopathy occurs in 1-7 infants per 1,000 live-term births. ${ }^{1}$ Its etiologies and associated risk factors include (i) antepartum events (reported in about $25 \%$ of affected term newborns), such as maternal hypotension, maternal thyroid disease, infertility treatment; (ii) intrapartum events (reported in less than $10 \%$ of cases), such as abruption of placenta, forceps delivery, breech presentation, maternal fever; and (iii) postnatal events (reported in less than $10 \%$ of cases), such as severe respiratory distress, sepsis, or shock. Despite the heterogeneous etiologies, hypoxic-ischemic encephalopathy (HIE) is considered causative in $50-75 \%$ of term newborns with neonatal encephalopathy. However, the majority of term newborns with neonatal encephalopathy attributed to HIE do not have a history of hypoxia or ischemia during the perinatal period. ${ }^{1,2}$ In fact, there are no identifiable risk factors in more than half of term newborns with neonatal encephalopathy. ${ }^{1,2}$

The severity of neonatal encephalopathy is on a spectrum with death in 15-20\% of affected term newborns. Between 65 to $98 \%$ of the newborns with moderate to severe neonatal encephalopathy develop cerebral palsy, characterized by impaired motor function. ${ }^{1-3}$

To date, the genetic causes of neonatal encephalopathy have not been investigated. However, characterizing the genetic contributors of neonatal encephalopathy will probably increase our understanding of neonatal brain injury in the term newborn and also, in time, enable us to develop new treatment modalities using large-scale drug screening based on the underlying genetic defect. To identify underlying genetic defects, we applied whole-exome sequencing (WES) in

\footnotetext{
${ }^{1}$ Genetics and Genome Biology Program, Research Institute, The Hospital for Sick Children, Toronto, Ontario, Canada; ${ }^{2}$ Division of Clinical and Metabolic Genetics, Department of Paediatrics, The Hospital for Sick Children, University of Toronto, Toronto, Ontario, Canada; ${ }^{3}$ Division of Neonatology, Department of Paediatrics, The Hospital for Sick Children, University of Toronto, Toronto, Ontario, Canada; ${ }^{4}$ Division of Neurology, Department of Pediatrics, The Hospital for Sick Children, University of Toronto, Toronto, Ontario, Canada; ${ }^{5}$ Division of Cell Proliferation, ART, Graduate School of Medicine, Tohoku University, Sendai, Japan; ${ }^{6}$ Department of Health Chemistry, Hoshi University, Tokyo, Japan; ${ }^{7}$ Department of Pediatrics, Graduate School of Medicine, Gifu University, Gifu, Japan; ${ }^{8}$ Genome Diagnostics, Department of Paediatric Laboratory Medicine, The Hospital for Sick Children, Toronto, Ontario, Canada; ${ }^{9}$ Institute of Medical Sciences, University of Toronto, Toronto, Onatrio, Canada. Correspondence: Saadet Mercimek-Andrews (saadet.andrews@sickkids.ca)
} 
term newborns with neonatal encephalopathy as an exploratory prospective cohort study.

\section{MATERIALS AND METHODS}

\section{Patients}

This study is approved by the institutional research ethics board (Approval \#100032244). Inclusion criteria were neonatal encephalopathy and/or prenatal or neonatal onset seizures or abnormal brain magnetic resonance imaging (MRI) features (such as edema, white matter and/or gray matter signal abnormalities, or hemorrhage) without history of perinatal asphyxia. Exclusion criteria were preterm delivery $(<37$ weeks gestation) and a sentinel hypoxic event occurring immediately before or during labor. Two of our coauthors and study team members (D.W. and V.C.), as well as Steven Miller (expert in neonatal encephalopathy and HIE), were in the circle of clinical care for all the patients with neonatal encephalopathy. They screened patients according to our inclusion and exclusion criteria and informed the research team of their selections for enrollment in the research study. Term newborns with neonatal encephalopathy meeting the inclusion criteria were enrolled after informed consent was obtained.

\section{WES}

DNA for WES was extracted from peripheral blood from index cases and their parents and stored at $-80^{\circ} \mathrm{C}$. WES was performed on patients and parents at the Centre for Applied Genomics, using the Illumina HiSeq 2000/2500 (Illumina, San Diego, CA, USA) following enrichment with the Agilent SureSelect Human All Exon kit V4 50-Mb (Agilent, Santa Clara, CA, USA). CASAVA (Illumina) was used for base calling and raw reads were mapped to the reference human genome (UCSC Genome Browser-hg19; University of California, Santa Cruz, CA, USA) using BWA version 0.6.5a. Duplicate pair end reads were removed using MarkDuplicates (Picard tools version 1.35; Broad Institute, Cambridge, MA, USA). The duplicate-free alignments were refined using local realignment and base quality score recalibration with GATK version1.0.5506 (Broad Institute). Variant calling was performed using the recommended best practices of GATK. ${ }^{4}$ Variant annotation and prioritization was performed using a previously described pipeline. ${ }^{5}$ Briefly, rare sequence variants, with frequency of occurrence $<1 \%$ in the National Heart, Lung, and Blood Institute exome server and 1000 Genomes control databases, the Exome Variant Server (EVS) database (http://evs.gs.washington.edu/EVS/PopStatsServlet? searchBy $=$ Gene + Hugotarget $=\mathrm{GATMx}=0 \mathrm{y}=0$ ), and the Exome Aggregation Consortium (ExAC) Broswer Beta dabase (Broad Institute) (URL: http://exac.broadinstitute.org/), were filtered for further analysis. Further prioritization was focused on rare variants including loss of function (frameshift, nonsense, and splice-site mutations), homozygous missense, and/or relevant known disease genes from the Online Mendelian Inheritance in Man database (OMIM). To determine whether missense variants were predicted to be deleterious, the predictors Sorting Intolerant From Tolerant
(SIFT) (http://sift.jcvi.org), ${ }^{6}$ Polymorphism Phenotyping 2 (PolyPhen-2) (http://genetics.bwh.harvard.edu/pph2/), and the Variant Effect Predictor were used. ${ }^{8}$ Candidates were chosen based on the following criteria: SIFT score, PolyPhen2 score, QualByDepth (QD: variant confidence from the QUAL field/unfiltered depth) $>5$, phylopMam $>2$, and phylopVert $>4$ (conservation in mammals and vertebrates respectively); CADD_phred score $>10$. Genes localized to the brain, with known neurological function, or clinically reported genes were chosen for validation and segregation in the patients and parents.

\section{Sanger sequencing of candidate variants}

Primers were designed to span a minimum of $100 \mathrm{bp}$ upstream and downstream of the variant of interest in the candidate gene. Genomic DNA from patients and parents was sequenced using Taq DNA Polymerase (ThermoFisher Scientific, \#18038067; Waltham, MA, USA), standard buffer and protocols. ${ }^{9}$ The polymerase chain reaction (PCR) product was purified using Nucleospin Gel and PCR Clean-up kit (Macherey-Nagel, Duren, Germany). Sequencing was performed according to our institution's sequencing methods. Sanger sequencing further confirmed nonsynonymous, nonSNP changes in patient and parents. All candidates with a known OMIM disease were confirmed in a clinical molecular diagnostic laboratory.

\section{Functional analysis}

The effects of identified variants on protein function for two genes, CULAB and AACS, were characterized and are briefly described below.

\section{CULAB variant functional analysis}

Lymphoblasts were lysed in a radioimmunoprecipitation buffer (50 mM Tris-HCl (pH 8.0), $0.1 \%$ SDS, $150 \mathrm{mM} \mathrm{NaCl}$, $1 \%$ Nonidet P-40, $0.5 \%$ sodium deoxycholate, protease and phosphatase inhibitor cocktail) at $4{ }^{\circ} \mathrm{C}$ for $10 \mathrm{~min} .{ }^{10-13}$ Crude lysates were cleared by centrifugation at $20,000 \mathrm{~g}$ at $4{ }^{\circ} \mathrm{C}$ for $15 \mathrm{~min}$, and the resulting supernatants were subjected to sodium dodecyl sulfate polyacrylamide gel electrophoresis (SDS-PAGE), followed by immunoblot analysis. The relative band intensity of CUL4B (normalized with Tubulin) was calculated with Image J software. Anti-CUL4B (HPA011880) and anti-Tubulin (T6074) antibodies were purchased from Sigma (Seattle, WA, USA). Anti-CUL4A (ab34897) antibody was from Abcam (Cambridge, UK). Anti-DDB1 antibody was a gift from Yue Xiong (University of North Carolina at Chapel Hill).

HEK293T cells transfected with an expression vector encoding myc-tagged either wild-type or mutant CUL4B (N211S or T231I) were treated with cycloheximide $(25 \mu \mathrm{g} / \mathrm{ml})$ for 0 to $6 \mathrm{~h}$, and then lysed with radioimmunoprecipitation assay buffer (50 mM Tris (pH 8.0), 0.1\% SDS, $150 \mathrm{mM} \mathrm{NaCl}$, $1 \%$ Nonidet P-40, $0.5 \%$ sodium deoxycholate, and protease inhibitor cocktail) at $4{ }^{\circ} \mathrm{C}$ for $10 \mathrm{~min}$. Crude lysates were cleared by centrifugation at $20,000 \mathrm{~g}$ at $4{ }^{\circ} \mathrm{C}$ for $15 \mathrm{~min}$, and 
supernatants were subjected to immunoblot analysis. The relative band intensity of CUL4B (normalized with Tubulin) was calculated with Image J software. Anti-Myc (sc-40) antibody was purchased from Santa Cruz Biotechnology (Dallas, TX, USA). Anti-Tubulin (T6074) antibody was from Sigma.

HEK293T cells transfected with an expression vector encoding myc-tagged either wild-type or mutant CUL4B (or the vector as a control) were washed with phosphatebuffered saline and lysed in an NP-40 lysis buffer (0.5\% Nonidet P-40, $50 \mathrm{mM}$ Tris ( $\mathrm{pH} 7.5$ ), $150 \mathrm{mM} \mathrm{NaCl}$, $10 \%$ glycerol and protease inhibitor cocktail) at $4{ }^{\circ} \mathrm{C}$ for $5 \mathrm{~min}$. Crude lysates were cleared by centrifugation at $20,000 \mathrm{~g}$ at $4{ }^{\circ} \mathrm{C}$ for $15 \mathrm{~min}$, and supernatants were incubated with Dynabeads protein G (Thermo Fisher Scientific) which was preconjugated with Myc antibody. The immunocomplexes were washed three times with a wash buffer $(0.1 \%$ Triton X-100, $10 \%$ glycerol in phosphate-buffered saline) and then subjected to SDS-PAGE, followed by immunoblot. Anti-Myc (sc-40) and anti-CSN5 (sc-13157) antibodies were purchased from Santa Cruz Biotechnology. Anti-DDB1 and anti-ROC1 antibodies were gifts from Yue Xiong (University of North Carolina at Chapel Hill, NC, USA).

\section{AACS variant functional analysis}

Antibody. Rabbit polyclonal antibodies were raised against the keyhole limpet hemocyanin-conjugated polypeptide MSKEERPGREEILEC (amino acids 1-15) of human AACS by Sigma-Aldrich (St Louis, MO, USA). The antibodies were affinity-purified using protein $\mathrm{G}$ sepharose and concentrated by ultrafiltration through an Amicon Ultra 30K device (Merck Millipore, Billerica, MA, USA).

Constructs. For subcloning into the Hind III/Kpn I restriction sites of pCMV-(DYKDDDDK)-N (Clontech Laboratories, Mountain View, CA, USA), the cDNA encoding human AACS was amplified using the forward primer 5'-AAGC TTATGTCCAAGGAGGAGCGCCC- $3^{\prime}$ and the reverse primer 5'-GGTACCTCAGAAGCCCTGCAGCTCAG-3' from cDNA of Hep G2 cells. The following double-stranded oligonucleotides with mutations were used as primers: $5^{\prime}-$ TAC GCTCAACAGCAAGAAAGTGG-3' for G632S (the underlined nucleotides encode the mutated residues). The insert was sequenced and was identical to human AACS (accession number NM_023928). The FLAG (DYKDDDDK)-hAACS ( $\mathrm{N}$ terminally FLAG-tagged human AACS) vectors $(1 \mu \mathrm{g})$ were transfected into the HEK293 Cell line. After 2 days of transfection, the cells were harvested in cell lysis buffer (10 mM Tris-HCl pH7.4, 1\% NP40, $150 \mathrm{mM} \mathrm{NaCl}$, and $0.5 \mathrm{mM}$ EDTA). AACS activity in $50 \mu \mathrm{g}$ of protein extracts was assayed by measuring acetyl-CoA formation according to a method previously reported by Hasegawa et al. ${ }^{14}$

\section{RESULTS}

\section{Patients}

The neonatal intensive care unit in our institution is a tertiary care center for term newborns with neonatal encephalopathy. During our study period of April 2013 to December 2016, there were 2,984 admissions to our neonatal intensive care unit. We do not have any registries giving the exact number of patients admitted with neonatal encephalopathy during the study period. However, between July 2014 and December 2016, using a database generated for Quality and Risk Management for a Neonatal Neurocritical Care Database (maintained by D.W.), we identified 270 patients admitted with neonatal encephalopathy to our neonatal intensive care unit. There were 176 patients with HIE, 15 patients with stroke, 13 patients with congenital brain malformation, 8 patients with meningitis, and two patients with neuromuscular disorders, who did not fulfill our inclusion criteria. Of the remaining 56 patients, 11 died prior to screening for our study. Some of the patients did not have both parents available for our WES trio (patient and both parents) study, and so were not included. Our estimated enrollment rate is $50 \%$ of patients with neonatal encephalopathy who fulfilled our inclusion criteria during our study period. Between April 2013 and December 2016, 19 patients and families fulfilling the inclusion criteria signed the consent forms to be included in the study during their admission to the neonatal intensive care unit at the time of their first symptom. Five patients were excluded for the following reasons: (i) one family withdrew their consent after completion of WES and data analysis; (ii) one patient received a clinical diagnosis prior to WES after achieving seizure freedom on pyridoxine therapy and being diagnosed with pyridoxine-dependent epilepsy caused by pathogenic variants in $A L D H 7 A 1$; (iii) one patient was diagnosed with viral encephalitis based on the response to antiviral therapy; and (iv) two families signed the consent forms but the parents did not provide blood samples for WES. The remaining 14 patients -4 males and 10 femalesunderwent WES. The average age of onset was $2.1 \pm 2.1 \mathrm{SD}$ days (range 10 min to 6 days). One patient passed away at the age of 6 days.

Demographics, clinical features, brain MRI and electroencephalography (EEG) results of all study patients are summarized in Table 1. All patients had neonatal encephalopathy and seizures. Pregnancy was remarkable for gestational diabetes mellitus in four mothers and oligohydramnios in two mothers. Three patients had hypoglycemia. Brain MRI features included diffuse symmetrical diffusion restriction, hemorrhages, an increased signal intensity in bilateral basal ganglia. Magnetic resonance spectroscopy showed a lactate peak in four patients. All patients had sharp waves and/or discontinuous background in EEG (Table 1).

\section{WES and functional analysis}

WES was performed on a trio (patient and both parents) in 14 patients. In seven patients we identified candidate variants and confirmed segregation in patients and parents 
Table 1 Clinical features, EEG findings, brain MRI features of patients enrolled into the study and their WES results summarized

\begin{tabular}{|c|c|c|c|c|}
\hline $\begin{array}{l}\text { Patient ID/sex/age } \\
\text { of onset }\end{array}$ & $\begin{array}{l}\text { Pregnancy/delivery/Apgar scores/clinical } \\
\text { features at presentation }\end{array}$ & Initial EEG & Brain MRI/MRS (age) & Candidate variant, gene, inheritance pattern \\
\hline NE_NS 001/M/ 2 days & $\begin{array}{l}\text { Oligohydramnios/planned C-section/9 and } \\
\text { 9/poor feeding, hypotonia, lip smacking, } \\
\text { encephalopathy, hypoglycemia }\end{array}$ & $\begin{array}{l}\text { Excessive background discontinuity, bilateral } \\
\text { independent sharp transients }\end{array}$ & $\begin{array}{l}\text { Global cortical and bilateral putamen diffusion restriction, } \\
\text { diffuse WM edema/prominent lactate peak ( } 4 \text { days) }\end{array}$ & $\begin{array}{l}\text { Hemizygous, novel, c.632A> G (p.Asn211Ser) in } \\
\text { CUL4B/X-linked, maternal }\end{array}$ \\
\hline NE_NS 002/F/2 days & $\begin{array}{l}\text { Unremarkable/vaginal forceps } / 3,5 \text {, and } 7 / a p n e a, \\
\text { desaturations at delivery, right-sided seizures }\end{array}$ & $\begin{array}{l}\text { Discontinuous background, multifocal } \\
\text { sharp waves }\end{array}$ & $\begin{array}{l}\text { Bilateral diffusion restriction in putamen, thalamus, } \\
\text { hippocampi, corticospinal tracts, cerebral peduncles, } \\
\text { dorsal pons/large lactate peak ( } 4 \text { days) }\end{array}$ & None \\
\hline $\begin{array}{l}\text { NE_NS 004/M/3 days } \\
\text { (died } 6 \text { days) }\end{array}$ & $\begin{array}{l}\text { Unremarkable/not known discharged } 24 \mathrm{~h} / \\
\text { lethargy, fever, poor feeding, focal seizures, } \\
\text { hypoglycemia }\end{array}$ & $\begin{array}{l}\text { Discontinuous background, multiple } \\
\text { multifocal subclinical seizures originating } \\
\text { from bilateral temporo-occipital head region }\end{array}$ & $\begin{array}{l}\text { Diffuse bilateral symmetrical diffusion restriction of the } \\
\text { cerebral cortex and basal ganglia, diffuse WM edema, } \\
\text { massive cerebral injury/lactate peak ( } 5 \text { days) }\end{array}$ & None \\
\hline NE_NS 005/F/36 h & $\begin{array}{l}\text { Unremarkable/C-section for fetal heart rate } \\
\text { decelerations/9 and 9/apnea, cyanosis, } \\
\text { GTS, lip smacking }\end{array}$ & $\begin{array}{l}\text { Burst suppression, ictal EEG with generalized } \\
\text { high-amplitude spike and sharp waves }\end{array}$ & $\begin{array}{l}\text { Inferior basal ganglia hyperintensity in T1 weighted image/ } \\
\text { NP ( } 6 \text { days) }\end{array}$ & $\begin{array}{l}\text { Heterozygous novel de novo c.634G }>\mathrm{T}(\mathrm{p} \text {. } \\
\text { Asp212Tyr) in KCNQ2/AD }\end{array}$ \\
\hline NE_NS 007/F/10 min & $\begin{array}{l}\text { Unremarkable/vaginal delivery/7 and 7/ } \\
\text { hypotonia, apnea, GTS }\end{array}$ & $\begin{array}{l}\text { Discontinuous background, bilateral negative } \\
\text { rolandic sharp waves }\end{array}$ & $\begin{array}{l}\text { Bilateral subdural, subarachnoid, and intraventricular } \\
\text { hemorrhages and anterolateral temporal ischemic } \\
\text { changes, diffuse WM edema/normal ( } 2 \text { days) }\end{array}$ & None \\
\hline NE_NS 008/M/12 h & $\begin{array}{l}\text { Unremarkable/vaginal vacuum } / 3 \text { and 6/eye } \\
\text { blinking, bicycling, GTS, apnea }\end{array}$ & $\begin{array}{l}\text { Discontinuous background, intermittent } \\
\text { sharp waves }\end{array}$ & $\begin{array}{l}\text { Diffuse brain edema with effacement of ventricular lumen } \\
\text { and pericerebral space/small lactate peak ( } 1 \text { day) }\end{array}$ & None \\
\hline NE_NS 009/F/8 h & Unremarkable/vaginal vacuum/8 and 9/GTCS & $\begin{array}{l}\text { Discontinuous background, frequent } \\
\text { temporal sharp waves }\end{array}$ & $\begin{array}{l}\text { Bilateral cortical T1 hyperintensity in posterior temporal } \\
\text { and perisylvian gyri, T2 hyperintensity in thalami/normal } \\
\text { ( } 7 \text { days) }\end{array}$ & $\begin{array}{l}\text { Homozygous c. } 1894 \mathrm{G}>\mathrm{A} \text { (p.Gly632Ser) in } \\
\text { AACS/AR/parents heterozygous }\end{array}$ \\
\hline $\begin{array}{l}\text { NE_NS 010/M/at } \\
\text { birth }\end{array}$ & $\begin{array}{l}\text { Gestational DM/vaginal/0, 0, and 8/poor tone } \\
\text { and apnea at birth, mild hypoxic-ischemic } \\
\text { encephalopathy, GTCS } 6 \text { days }\end{array}$ & $\begin{array}{l}\text { Discontinuous background, bilateral } t \\
\text { emporal sharp waves }\end{array}$ & $\begin{array}{l}\text { Bilateral increased signal in frontal and peritrigonal WM in } \\
T 1 / \text { normal (8 days) }\end{array}$ & $\begin{array}{l}\text { Heterozygous de novo, novel c.118G > A ( } p \text {. } \\
\text { Gly40Arg) in GNAO1/AD }\end{array}$ \\
\hline NE_NS 011/F/6 h & $\begin{array}{l}\text { Gestational DM/C-section for failure to progress/ } \\
7 \text { and 9/hypoglycemia (1 h) GTCS, apnea }\end{array}$ & $\begin{array}{l}\text { Discontinuous background, burst suppression } \\
\text { pattern, central midline sharp waves }\end{array}$ & $\begin{array}{l}\text { Bilateral diffuse cortical and subcortical WM diffusion } \\
\text { restriction most prominent in occipital lobes/normal ( } 1 \text { day) }\end{array}$ & None \\
\hline NE_NS 015/F/6 days & $\begin{array}{l}\text { Unremarkable/vaginal/6 and 8/apnea, focal } \\
\text { tonic seizures }\end{array}$ & $\begin{array}{l}\text { Discontinuous background, bilateral central } \\
\text { temporal sharp waves }\end{array}$ & $\begin{array}{l}\text { Bilateral frontoparietal subcortical WM T2 hyperintensity/ } \\
\text { normal ( } 9 \text { days) }\end{array}$ & $\begin{array}{l}\text { Heterozygous, novel, de novo, c.4633A> G } \\
\text { (p.Met1545Val) in SCN2A/AD }\end{array}$ \\
\hline NE_NS 016/F/21 h & Oligohydramnios/vaginal/8 and 9/focal seizures & $\begin{array}{l}\text { Discontinuous background, bilateral central } \\
\text { and temporal rolandic sharp waves }\end{array}$ & $\begin{array}{l}\text { Bilateral cortical subcortical, deep WM and basal ganglia } \\
\text { diffusion restriction/normal ( } 2 \text { days) }\end{array}$ & None \\
\hline NE_NS 017/F/24 h & $\begin{array}{l}\text { Gestational DM/vaginal delivery/7 and } \\
\text { 8/hypotonia, focal seizures and GTCS }\end{array}$ & $\begin{array}{l}\text { Discontinuous background, bilateral central } \\
\text { temporal sharp waves }\end{array}$ & $\begin{array}{l}\text { Bilateral diffuse cortical, corpus callosum splenium, } \\
\text { thalamus and posterior limb internal capsules diffusion } \\
\text { restriction/normal ( } 2 \text { days) }\end{array}$ & $\begin{array}{l}\text { Heterozygous, novel, de novo, c.1692G > A (p. } \\
\text { Met564lle) in RAl1/AD }\end{array}$ \\
\hline NE_NS018/F/2 days & $\begin{array}{l}\text { Unremarkable/vaginal delivery term/7 and } \\
\text { 9/GTCS }\end{array}$ & Bilateral central temporal sharp waves & $\begin{array}{l}\text { Symmetrical diffusion restriction in frontal, perirolandic, } \\
\text { and occipital white matter, increased T2 signal in medial } \\
\text { thalami/normal ( } 3 \text { days) }\end{array}$ & None \\
\hline NE_NS019/F/1 day & $\begin{array}{l}\text { Unremarkable/C-section/8 and 9/focal } \\
\text { and GTCS }\end{array}$ & $\begin{array}{l}\text { Multifocal frequent sharp waves, } \\
\text { discontinuous background }\end{array}$ & $\begin{array}{l}\text { Diffuse symmetrical white matter diffusion restriction } \\
\text { ( } 2 \text { days), progressive diffusion restriction in white matter, } \\
\text { brainstem, cerebellar white matter ( } 9 \text { days)/increased } \\
\text { glycine peak }\end{array}$ & $\begin{array}{l}\text { Compound heterozygous c.757G >C (p. } \\
\text { Ala253Pro, maternal)/c.708T > G (p.His236Gln, } \\
\text { paternal) in LIAS/AR }\end{array}$ \\
\hline
\end{tabular}




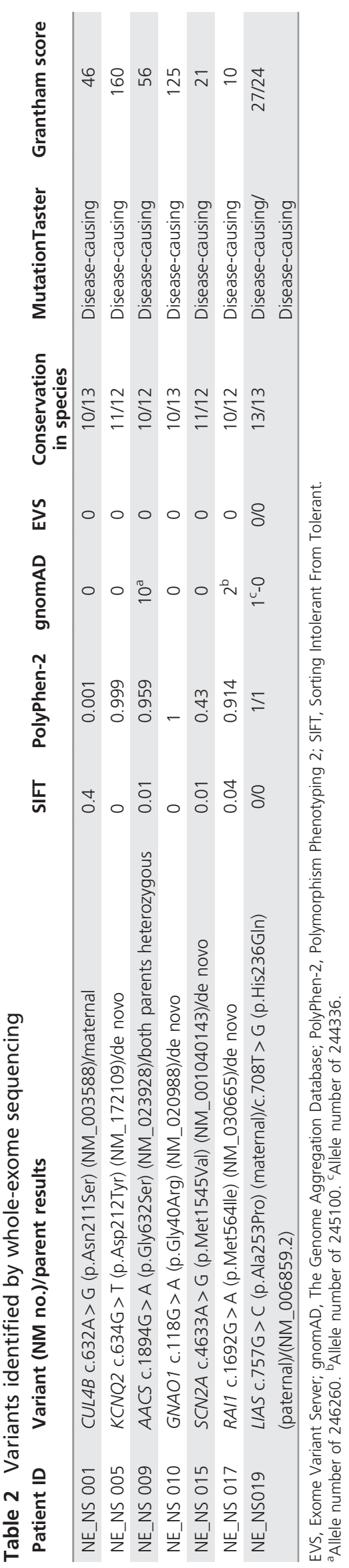

with Sanger sequencing. In silico analyses of likely pathogenic variants in those seven patients are summarized in Table 2. We confirmed a clinical genetic diagnosis in four patients in a Clinical Laboratory Improvement Amendments-certified laboratory, including SCN2A-, KCNQ2-, and GNAO1associated epileptic encephalopathies and lipoic acid synthetase deficiency caused by biallelic likely pathogenic variants in LIAS. The SCN2A p.Met1545Val, KCNQ2 p.Asp212Tyr, and GNAO1 p.Gly40Arg de novo variants were the cause of neonatal encephalopathy and seizures in these patients.

In three patients we identified variants in two known genes, CULAB and RAI1, and a novel gene, AACS. The functional analysis of the hemizygous p.Asn211Ser variant in CUL4B showed a decreased CUL4B protein level, confirming the pathogenicity (Figure 1a-c). A homozygous p.Gly632Ser variant in AACS encoding acetoacetyl-CoA synthetase (AACS) was identified in one patient as a novel candidate gene. The first cousin parents were heterozygous carriers for the same variant. We found normal AACS activity in the homogenates from cells overexpressing AACS p.Gly632Ser variant, similar to that in wild-type cells (Figure 2a). Immunoblot of p.Gly632Ser variant showed the same amount of AACS protein as in wildtype (Figure 2a and b). We concluded that this variant is not pathogenic. We were not able to perform functional analysis for the RAI1 p.Met564Ile variant.

\section{DISCUSSION}

We utilized WES in cases of neonatal encephalopathy and/or seizures in a prospective cohort study to investigate underlying causes of neonatal encephalopathy. We identified three known genes causing neonatal epileptic encephalopathy: KCNQ2, SCN2A, and GNAO1. We also identified a new patient with lipoic acid synthetase deficiency caused by biallelic likely pathogenic variants in LIAS mimicking neuroimaging features of HIE. We identified a missense variant in CULAB in a male, confirmed to be pathogenic by functional analysis, as the cause of global developmental delay and seizures in this patient. The genetic diagnostic yield of WES was $36 \%$ (5 out of 14) for neonatal encephalopathy and/or neonatal seizures in our prospective cohort study. Additionally, we found a de novo likely pathogenic variant in RAI1 in a 10-month-old girl with microcephaly and global developmental delay with no behavioral problems or sleep disturbances. Likely pathogenic variants in RAI1 cause SmithMagenis syndrome, which is a neurodevelopmental disorder characterized by dysmorphic features, intellectual disability, and sleep disturbances. We are not certain if the likely pathogenic variant in RAI1 in our patient would explain her phenotype, in the absence of typical phenotypic features of Smith-Magenis syndrome. In another patient, we had high suspicion of an AACS deficiency causing a novel disease, based on the prediction tools, using in silico analysis. AACS is an important enzyme for acetyl-CoA production and lipid synthesis. It is required for normal neuronal development, and is expressed strongly in the human and rat brains. ${ }^{15}$ However, functional analysis of the patient's AACS 

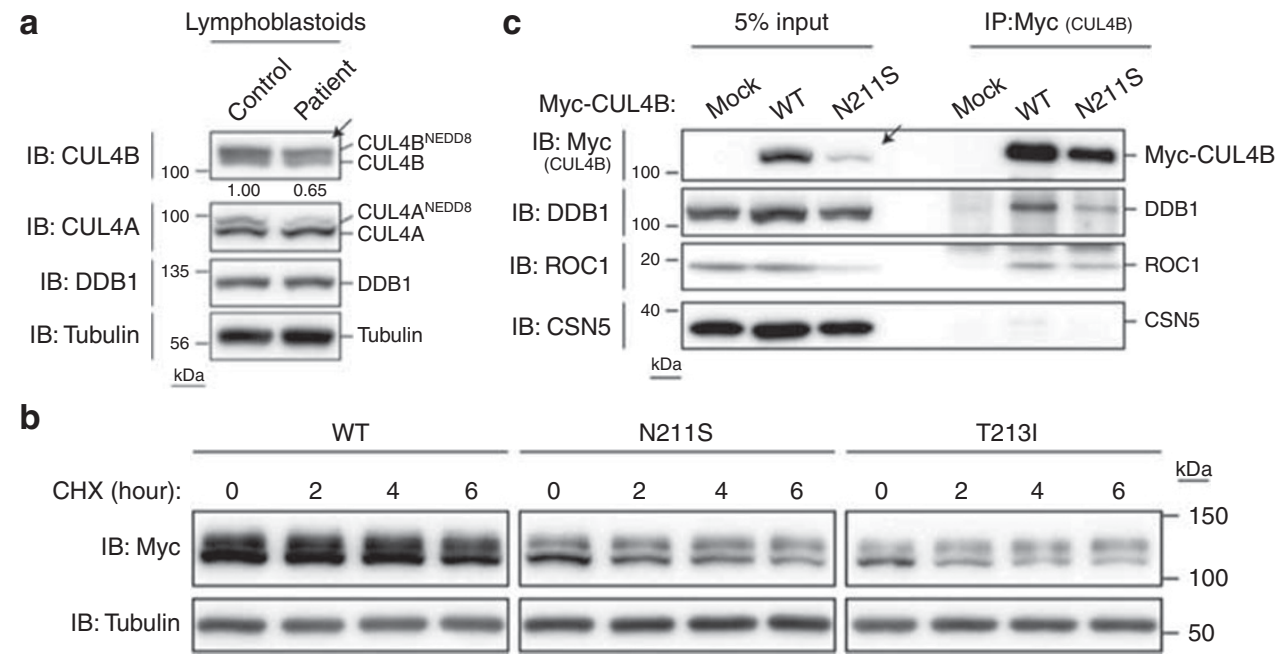

Figure 1 Functional analysis of p.Asn211Ser CUL4B variant. (a) Reduced protein level of CUL4B in patient lymphoblasts with p.Asn211Ser CUL4B variant compared with control lymphoblasts (indicated by the arrow). (b) Reduced stability of p.Asn211Ser CUL4B variant. (c) Formation of ubiquitin ligase complex by p.Asn211Ser CUL4B variant. The arrow indicates reduced expression of p.Asn211Ser CUL4B compared with wild-type CUL4B. CHX, cycloheximide (25 $\mu \mathrm{g} / \mathrm{ml})$; CSN5, COP9 signalosome subunit 5; CUL4ANEDD8, NEDD8-modified CUL4A; CUL4BNEDD8, NEDD8-modified CUL4B; DDB1, damage-specific DNA binding protein 1; IP, immunoprecipitation; ROC1, regulator of cullins-1.

a



b

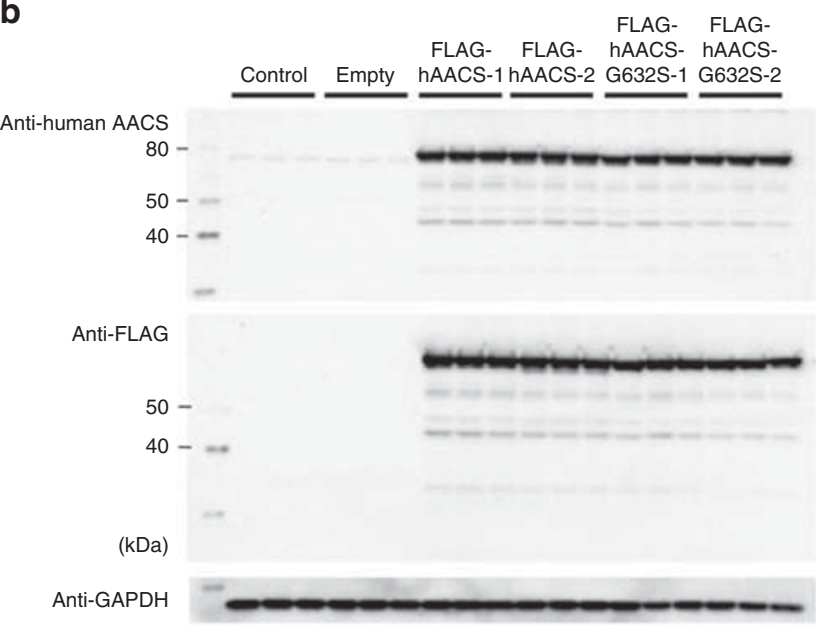

Figure 2 Activity of AACS G632S variant. (a) FLAG-hAACS-1 and -2 vectors or FLAG-hAACS-G632S-1 and -2 vectors were isolated from the separate bacterial colonies. The empty FLAG-hAACS or FLAG-hAACS-G632S vectors were transfected into the HEK 293 cell line. After 2 days of culture, the cells were harvested in the TNE buffer. AACS activity in $50 \mu \mathrm{g}$ of protein extracts was assayed by measuring acetyl-CoA formation, as described in "Materials and Methods." Data are representative of three independent experiments. Error bars indicate the SD (b) Each cell lysate (7.5 $\mathrm{g}$ of protein) was subjected to western blotting to detect human AACS, FLAG, and GAPDH (glyceraldehyde 3-phosphate dehydrogenase).

p.Gly632Ser missense variant revealed normal AACS activity, which excludes the possibility of AACS deficiency. This illustrates the importance of functional studies for missense variants to confirm pathogenicity in the process of identification of novel genetic disorders.

One of the purposes of our study was to investigate whether term newborns with neonatal encephalopathy have genetic defects of energy metabolism pathways that contribute to brain energy failure and neonatal brain injury at delivery, mimicking HIE without any history of hypoxia. We identified lipoic acid synthetase deficiency and severe brain damage without history of hypoxia in a patient. Biallelic likely pathogenic variants in LIAS, encoding lipoic acid synthetase, result in defective lipoic acid synthesis. Lipoic acid is a cofactor for pyruvate dehydrogenase, branched-chain ketoacid dehydrogenase, 2-ketoglutarate dehydrogenase, and 2 -oxoadipate dehydrogenase. All of these enzymes have key functions in mitochondrial energy metabolism and production of energy. Because of a lack of the cofactor lipoic acid, these enzymes are dysfunctional and cause decrease in energy 
production, contributing to lack of energy and hypoxic changes visible in brain MRI, mimicking HIE in our patient. To date, three patients have been reported with lipoic acid synthetase deficiency. ${ }^{16,17}$ All patients presented with elevated glycine levels. Our patient had also elevated plasma and cerebrospinal fluid glycine levels, with high cerebrospinal fluid to plasma glycine ratio $(0.1$; reference range $<0.02)$ with normal blood lactate levels consistent with the biochemical features of previously reported patients. We report the fourth patient with lipoic acid synthetase deficiency identified in this study. A small number of genetic disorders in energy or cofactor metabolism mimic the clinical and neuroimaging features of HIE, including cytochrome C oxidase deficiency, ${ }^{18}$ pyridoxine dependent epilepsy, ${ }^{19}$ molybdenum cofactor deficiency, ${ }^{20}$ glycogen storage disease type IV, ${ }^{21}$ and X-linked centronuclear myopathy. ${ }^{22}$ Lipoic acid synthetase deficiency should be included in the differential diagnosis of HIE without a history of hypoxia. Application of WES in patients with neonatal encephalopathy will probably identify more genetic disorders involving energy metabolism pathways contributing to the pathogenesis of term neonatal encephalopathy and will increase our understanding of severe neonatal brain damage without history of hypoxia.

A previous study assessed the diagnostic yield of WES in 20 acutely ill newborns, using targeted WES including 4,183 known disease-associated genes. ${ }^{23}$ Patients presented with hypotonia (six patients), seizures (four patients), multiple malformations (six patients), family history of the same complex clinical presentation (two patients), and consanguineous parents (three patients). ${ }^{23}$ Twelve patients were identified retrospectively and enrolled after discharge from the neonatal intensive care unit and eight patients were enrolled when they were still in the neonatal intensive care unit. A genetic diagnosis was confirmed in eight patients $(40 \%)$. Seizures were present in four patients $(25 \%)$ and only one of those received the diagnosis of SCN1A-associated epileptic encephalopathy. In our prospective cohort study, we targeted patients with neonatal encephalopathy and/or seizures and enrolled them at the time of their first symptom during their initial admission. We arrived at a genetic diagnosis in $36 \%$ of the patients for known disease-associated genes associated with phenotypes in our prospective cohort study. Sixty percent of our patients had one of the epileptic encephalopathy genes, whereas in the previous study only $12.5 \%$ of the patients had an epileptic encephalopathy gene. ${ }^{23}$ We also identified a patient with lipoic acid synthetase deficiency involving energy metabolism pathways causing neonatal encephalopathy and brain injury. To the best of our knowledge, our study is the first to enroll patients with neonatal encephalopathy at their first presentation to investigate underlying genetic causes.

Unfortunately, we did not identify any candidate genes in $50 \%$ of the patients and there was no confirmed genetic diagnosis in $64 \%$ of the patients. This could be associated with the limitations of WES, including (i) targeting only the exonic portion of the genome (approximately 1\%); (ii) requiring exon capture and enrichment strategies which introduce bias due to nonhomogeneous capture in all exons; (iii) difficulties with GC-rich or repetitive regions; and (iv) PCR-based enrichment strategies that suffer from general stochastic, template-switch, and polymerase errors. ${ }^{24}$ The advantages of WES are that it is less costly, with a higher depth of coverage and a smaller amount of data generation for analysis and diagnosis of known Mendelian diseases in clinical settings. ${ }^{25}$ Rapid whole-genome sequencing was applied to 16 newborns and infants in the neonatal (15 patients) or pediatric (1 patient) intensive care units, and 10 of those (62.5\%) received a genetic diagnosis. ${ }^{26}$ Only four of those patients were enrolled within the first 3 weeks of life. The diagnostic yield of whole-genome sequencing was about $50 \%$ higher than that in our study results. This is probably due to advantages of whole-genome sequencing, including (i) coverage of both exonic and intronic regions, (ii) more uniform and complete coverage of the genome, (iii) the ability to identify noncoding pathogenic variants, and (iv) detection of copy-number variants. ${ }^{27}$ Applying whole-genome sequencing to our patient population without a genetic diagnosis might increase the diagnostic yield in neonatal encephalopathy.

Likely pathogenic variants in CUL4B have been reported to be associated with intellectual disability (OMIM 300354). ${ }^{28}$ Ventriculomegaly, cerebral and cerebellar atrophy, white matter volume loss, and polymicrogyria have been reported in patients with likely pathogenic variants in CUL4B. ${ }^{10}$ The p.Asn211Ser variant in CUL4B showed a weak CSN5 immunoprecipitation and was unstable in cycloheximide treatment. Patient lymphoblast lysates with p.Asn211Ser variant showed a decreased CUL4B protein level similar to those found with the previously reported pathogenic missense variants. ${ }^{10}$ The p.Asn211Ser variant in CUL4B did not affect complex formation of CUL4B ubiquitin ligase, which was also reported previously for missense pathogenic variants. ${ }^{10}$ For these reasons, we conclude that the p.Asn211Ser variant in CUL4B results in instable CUL4B protein, confirming the pathogenicity. Our patient with the pathogenic CUL4B variant presented with oligohydramnios and neonatal encephalopathy and seizures at the age of 2 days, and had extensive brain damage showing in brain MRI in the neonatal period. The repeat brain MRI revealed cerebral atrophy, encephalomalacia, and diffuse thinning of the corpus callosum. Our patient's brain MRI features are at the severe end of the reported spectrum. ${ }^{10}$

Neuroimaging features of patients with KCNQ2 associated neonatal epileptic encephalopathy include increased signal intensities in basal ganglia, thalamus, and hippocampus, diffuse hypomyelination and a thin corpus callosum. ${ }^{29}$ Our patient had bilateral subdural, subarachnoid, and intraventricular hemorrhages and anterolateral temporal ischemic changes, as well as diffuse white matter edema observed in brain MRI. We think that the neuroimaging features of our patient expand the clinical spectrum of patients with KCNQ2-associated neonatal epileptic encephalopathy to include intracerebral bleeding. 
Recently the phenotype and genotype of 201 patients with SCN2A related disorders have been reported. ${ }^{30}$ About $35 \%$ of those patients presented with early infantile epileptic encephalopathy, with a seizure onset within the first 3 months of life. A patient with the p.Met1545Val variant was reported in that study, who presented with neonatal onset seizures at the age of 2 days, characterized by tonic and clonic and right and left migrating seizures with normal brain MRI. ${ }^{30}$ In our patient seizure onset was at the age of 6 days, characterized by apnea and focal tonic seizures. Our patient had increased T2 signal intensity in the bilateral frontoparietal subcortical white matter in the brain MRI. Neonatal onset epileptic encephalopathy is the unique feature in both patients with the same likely pathogenic missense variant.

Fewer than 30 patients have been reported with GNAO1associated encephalopathy. About half of those patients presented with seizures and the other half presented with movement disorder without seizures. ${ }^{31}$ We summarized all patients with GNAO1-associated epileptic encephalopathy (Supplementary Table 1 online)..$^{32-40}$ Seizure onset was in the neonatal period in approximately half of the patients and only two patients presented with seizures at birth. The main neuroimaging features were cerebral atrophy, delayed myelination, and a thin corpus callosum. Our patient had seizure onset at birth. He presented with abnormal neuroimaging features, including increased signal intensities in frontal and peritrigonal white matter in brain MRI at 8 days of age.

In summary, we report a 36\% diagnostic yield of WES in neonatal encephalopathy and/or seizures in a prospective cohort study. This study, although small, represents an important step in improving our understanding of neonatal encephalopathy. Whole-genome sequencing will probably increase the diagnostic yield of genetic disorders and help us to discover novel genetic disorders causing neonatal encephalopathy and brain damage. The identification of novel genetic disorders involving energy metabolism pathways might provide insights into the pathogenesis of neonatal encephalopathy and brain damage and help us to develop targeted neuroprotective treatment strategies that will ultimately improve health outcomes in term newborns with neonatal encephalopathy.

\section{SUPPLEMENTARY MATERIAL}

Supplementary material is linked to the online version of the paper at http://www.nature.com/gim

\section{ACKNOWLEDGMENTS}

This study is partly funded by the Division of Clinical and Metabolic Genetics and Centre for Genetic Medicine Starbucks Clinical Genetics Genomic Research Studentship Award to S.M.-A. and T.U.J.B. and a University of Toronto McLaughlin Accelerator Grant in Genomic Medicine MC-2013-08 to S.M.-A. S.M.-A. thanks Berge Minassian for his tremendous support in providing laboratory space for molecular genetic studies and encouragement in the development of S.M.-A.'s research program and his continuing guidance and mentorship. We are also grateful to the following people and groups: Peter (Peixiang) Wang for his guidance in molecular genetic methods; the parents and patients for their contributions to our research study; Sergio Pereira and the informatics staff at The Center for Applied Genomics, The Hospital for Sick Children, for WES and technical expertise; Steven Miller for his mentorship; Stacy Hewson, Ashley Wilson, and Duncan Westwood for obtaining patient consents, Garrett Bullivant for analyzing one patient's WES data; Andrew James for his involvement at the stage of research ethics board submission; the Genome Aggregation Database (gnomAD) and the groups that provided exome and genome variant data to this resource (a full list of contributing groups can be found at http://gnomad.broadinstitute.org/about); and the Exome Aggregation Consortium and the groups that provided exome variant data for comparison (a full list of contributing groups can be found at http://exac.broadinstitute.org/about).

\section{DISCLOSURE}

The authors declare no conflict of interest.

\section{REFERENCES}

1. Ferriero DM. Neonatal brain injury. N Engl J Med. 2004;351:1985-1995.

2. Volpe JJ. Neonatal encephalopathy: an inadequate term for hypoxicischemic encephalopathy. Ann Neurol 2012;72:156-166.

3. Kurinczuk JJ, White-Koning M, Badawi N. Epidemiology of neonatal encephalopathy and hypoxic-ischaemic encephalopathy. Early Hum Dev. 2010;86:329-338.

4. McKenna A, Hanna M, Banks E, et al. The Genome Analysis Toolkit: a MapReduce framework for analyzing next-generation DNA sequencing data. Genome Res 2010;20:1297-1303.

5. Tammimies K, Marshall CR, Walker S, et al. Molecular diagnostic yield of chromosomal microarray analysis and whole-exome sequencing in children with autism spectrum disorder. JAMA 2015:314:895.

6. Kumar P, Henikoff S, Ng PC. Predicting the effects of coding nonsynonymous variants on protein function using the SIFT algorithm. Nat Protoc. 2009;4:1073-1081.

7. Adzhubei IA, Schmidt S, Peshkin L, et al. A method and server for predicting damaging missense mutations. Nat Methods 2010;7: 248-249.

8. McLaren $W$, Pritchard $B$, Rios D, Chen $Y$, Flicek $P$, Cunningham $F$. Deriving the consequences of genomic variants with the Ensembl API and SNP Effect Predictor. Bioinformatics 2010;26:2069-2070.

9. Innis MA, Gelfand DH, Sninsky JJ, and White TJ (eds). PCR Protocols: A Guide to Methods and Applications. Academic Press: San Diego, CA, 1990.

10. Vulto-van Silfhout, Nakagawa T, Bahi-Buisson N, et al. Variants in CULAB are associated with cerebral malformations. Hum Mutat. 2015;36: 106-117.

11. Hu J, Zacharek S, He YJ, et al. WD40 protein FBW5 promotes ubiquitination of tumor suppressor TSC2 by DDB1-CUL4-ROC1 ligase. Genes Dev. 2008:22:866-871.

12. Nakagawa $T$, Xiong $Y$. X-linked mental retardation gene CUL $4 B$ targets ubiquitylation of $\mathrm{H} 3 \mathrm{~K} 4$ methyltransferase component WDR5 and regulates neuronal gene expression. Mol Cell. 2010;43:381-391.

13. Ohta T, Michel JJ, Schottelius AJ, Xiong Y. ROC 1, a homolog of APC11, represents a family of cullin partners with an associated ubiquitin ligase activity. Mol Cell. 1991;3:535-541.

14. Hasegawa S, Inoue D, Yamasaki M, et al. Site-specific cleavage of acetoacetyl-CoA synthetase by legumain. FEBS Lett 2016;590: 1592-1601.

15. Hasegawa S, Kume H, linuma S, Yamasaki M, Takahashi N, Fukui T. Acetoacetyl-CoA synthetase is essential for normal neuronal development. Biochem Biophys Res Commun. 2012;427:398-403.

16. Mayr JA, Zimmermann FA, Fauth $C$, et al. Lipoic acid synthetase deficiency causes neonatal-onset epilepsy, defective mitochondrial 
energy metabolism, and glycine elevation. Am J Hum Genet. 2011;89: 792-797.

17. Baker PR, Friederich MW, Swanson MA, et al. Variant non ketotic hyperglycinemia is caused by mutations in LIAS, BOLA3 and the novel gene GLRX5. Brain 2014;137(Pt 2):366-379.

18. Willis TA, Davidson J, Gray RG, Poulton K, Ramani P, Whitehouse W. Cytochrome oxidase deficiency presenting as birth asphyxia. Dev Med Child Neurol. 2000;42:414-417.

19. Mercimek-Mahmutoglu S, Horvath GA, Coulter-Mackie M, et al. Profound neonatal hypoglycemia and lactic acidosis caused by pyridoxine-dependent epilepsy. Pediatrics. 2012;129:e1368-e1372.

20. Topcu M, Coskun T, Haliloglu G, Saatci I. Molybdenum cofactor deficiency: report of three cases presenting as hypoxic-ischemic encephalopathy. J Child Neurol. 2001;16:264-270.

21. Escobar LF, Wagner S, Tucker M, Wareham J. Neonatal presentation of lethal neuromuscular glycogen storage disease type IV. J Perinatol 2012;32:810-813.

22. Braga SE, Gerber A, Meier C, et al. Severe neonatal asphyxia due to X-linked centronuclear myopathy. Eur J Pediatr. 1990;150:132-135.

23. Daoud H, Luco SM, Li R, et al. Next-generation sequencing for diagnosis of rare diseases in the neonatal intensive care unit. CMAJ. 2016;188: E254-60.

24. Meyts I, Bosch B, Bolze A, et al. Exome and genome sequencing for inborn errors of immunity. J Allergy Clin Immunol. 2016;138: 957-969.

25. Horak P, Fröhling S, Glimm H. Integrating next-generation sequencing into clinical oncology: strategies, promises and pitfalls. ESMO Open. 2016;1:e000094.

26. Soden SE, Saunders CJ, Willig LK, et al. Effectiveness of exome and genome sequencing guided by acuity of illness for diagnosis of neurodevelopmental disorders. Sci Trans/ Med 2014;6:265ra168.

27. Belkadi A, Bolze A, Itan $Y$, et al. Whole-genome sequencing is more powerful than whole-exome sequencing for detecting exome variants. Proc Natl Acad Sci. 2015;112:5473-5478.

28. Tarpey PS, Raymond FL, O'Meara S, et al. Mutations in CUL4B, which encodes a ubiquitin E3 ligase subunit, cause an X-linked mental retardation syndrome associated with aggressive outbursts, seizures, relative macrocephaly, central obesity, hypogonadism, pes cavus, and tremor. Am J Hum Genet. 2007;80:345-352.

29. Pisano $T$, Numis $A L$, Heavin $S B$, et al. Early and effective treatment of KCNQ2 encephalopathy. Epilepsia 2015;56:685-691.

30. Wolff M, Johannesen KM, Hedrich UB, et al. Genetic and phenotypic heterogeneity suggest therapeutic implications in SCN2A-related disorders. Brain; e-pub ahead of print, 4 March 2017.

31. Menke LA, Engelen M, Alders M, Odekerken VJJ, Baas F, Cobben JM. Recurrent GNAO1 mutations associated with developmental delay and a movement disorder. J Child Neurol. 2016;31:1598-1601.

32. Nakamura K, Kodera H, Akita T, et al. De novo mutations in GNAO1, encoding a $G \alpha O$ subunit of heterotrimeric $G$ proteins, cause epileptic encephalopathy. Am J Hum Genet. 2013;93:496-505.

33. Appenzeller S, Balling R, Barisic N, et al. De novo mutations in synaptic transmission genes including DNM1 cause epileptic encephalopathies. Am J Hum Genet. 2014;95:360-370.

34. Law C-Y, Chang ST-L, Cho SY, et al. Clinical whole-exome sequencing reveals a novel missense pathogenic variant of GNAO1 in a patient with infantile-onset epilepsy. Clin Chim Acta. 2015;451(Pt B):292-296.

35. Talvik I, Møller RS, Vaher M, et al. Clinical phenotype of de novo GNAO1 mutation. Child Neurol Open. 2015;2:2329048X1558371.

36. Saitsu H, Fukai R, Ben-Zeev B, et al. Phenotypic spectrum of GNAO1 variants: epileptic encephalopathy to involuntary movements with severe developmental delay. Eur J Hum Genet. 2016;24:129-134.

37. Marcé-Grau A, Dalton J, López-Pisón J, et al. GNAO1 encephalopathy: further delineation of a severe neurodevelopmental syndrome affecting females. Orphanet J Rare Dis. 2016;11:38.

38. Gawlinski P, Posmyk R, Gambin T, et al. PEHO syndrome may represent phenotypic expansion at the severe end of the early-onset encephalopathies. Pediatr Neurol 2016;60:83-87.

39. Epi4K Consortium. De novo mutations in SLC1A2 and CACNA1A are important causes of epileptic encephalopathies. Am J Hum Genet. 2016;99:287-298.

40. Arya R, Spaeth C, Gilbert DL, Leach JL, Holland KD. GNAO1-associated epileptic encephalopathy and movement disorders: c.607G > A variant represents a probable mutation hotspot with a distinct phenotype. Epileptic Disord 2017;19:67-75. 